\title{
Application of Joint Electro-Chemical Detection for Gas Insulated Switchgear Fault Diagnosis
}

\author{
Liping $\mathrm{Li}^{*}$, Ju Tang ${ }^{\dagger}$ and Yilu Liu**
}

\begin{abstract}
The integrity of the gas insulated switchgear (GIS) is vital to the safety of an entire power grid. However, there are some limitations on the techniques of detecting and diagnosing partial discharge (PD) induced by insulation defects in GIS. This paper proposes a joint electro-chemical detection method to resolve the problems of incomplete PD data source and also investigates a new unique fault diagnosis method to enhance the reliability of data processing. By employing ultra-high frequency method for online monitoring and the chemical method for detecting SF6 decomposition offline, the acquired data can form a more complete interpretation of PD signals. By utilizing DS evidence theory, the diagnostic results with tests on the four typical defects show the validity of the new fault diagnosis system. With higher accuracy and lower computation cost, the present research provides a promising way to make a more accurate decision in practical application.
\end{abstract}

Keywords: Gas insulated switchgear, Partial discharge, Fault diagnostic, DS evidence theory, Joint electro-chemical method

\section{Introduction}

Gas insulated switchgear (GIS) is a multipart equipment that combines busbars, disconnectors, circuit-breakers, instrument transformers, cable terminations and joints in an earthed enclosure filled with $\mathrm{SF}_{6}$ gas. GIS offers advantages such as higher reliability, less maintenance, and more compact size. However, service experience also indicates incidents of GIS failure in recent years [1]. The operation of GIS equipment does not only affect its own stability of functioning but also has a direct impact on the power grid, causing economic losses to industry and inconvenience to people's daily life. In the urgent need are the effective and efficient procedure of dielectric detection and diagnosis to accurately recognize the partial discharge (PD) induced by insulation defects before they lead to any kind of damage.

There are a variety of diagnostic approaches based on pulse current, acoustic, chemical, and ultra-high frequency (UHF) detection techniques [2-10]. However, each of these approaches can be performed effectively to some extent with its own limitations. Furthermore, none of these detection methods can be used independently to completely eliminate the interface from the surrounding because PD signals obtained via each detection method contain only certain aspects of information. The pulse current technique has a weak capacity in resisting interference. Being easily

$\dagger$ Corresponding Author: Dept. of Electrical and Electronic Engineering, Chongqing University, China. (cqtangju@cqu.edu.cn)

* Dept. of Electrical and Electronic Engineering, Chongqing University, China. (lee.liping01@gmail.com)

** Dept. of Electrical and Electronic Engineering, University of Tennessee (Knoxville), U.S.A .(liu@utk.edu)

Received: December 28, 2014 ; Accepted: February 27, 2015 confounded by noise, the ultrasonic detection technique can result in inaccurate PD detection and recognition. A major limitation encountered by UHF is the problem of discharge calibration. Lastly, more studies are needed for the chemical method of detecting $\mathrm{SF}_{6}$ decomposition under PDs as it is a relatively new area. On the other hand, the diagnostic algorithms have not been completely resolved, despite their use for fault diagnosis, such as artificial neural networks (NN) [11], support vector machine (SVM) [12]. The diagnostic algorithms are quite inconvenient for artificial diagnosis and onsite quick diagnosis, resulting in limited applicability for field use.

In order to acquire a complete perceptive interpretation of PD signals, a joint electro-chemical detection method is proposed in this paper. For GIS population in service, ultra-high frequency (UHF) detection method is most frequently employed for online monitoring due to their higher reliability and sensitivity. As for the chemical method of detecting the SF6 decomposition products in GIS, it is an indispensable way for offline measurement since it's rarely affected by noise and electromagnetic interferences. The two complementary aspects of PD signals can provide rich electromagnetic and sufficient chemical information for fault diagnosis. Pertaining to these aspects, a new diagnostic method based on Dempster-Shafer (DS) evidence theory $[13,14]$ is proposed to enhance accuracy and ability of error tolerance.

The rest of the paper is organized as follow: Section 2 briefly introduces experimental setup and procedure for the joint electro-chemical detection method. Section 3 outlines the classic clustering algorithm for fault diagnosis under UHF method and chemical method respectively. Following the description of the principles of DS evidence theory, 
Section 4 illustrates a new diagnostic approach with the joint detection method. Conclusions are noted in Section 5.

\section{Joint Electro-Chemical Detection Method}

Service experience indicates that the main defects in real GIS in service include four typical ones, namely, metallic protrusion, free metallic particle, contamination on the surface of the spacer, and the gap at electrode/epoxy interface [1]. These four typical insulation defects are established for UHF and chemical detection.

\subsection{UHF online detection}

Most commonly used for online detection of PD, a UHF sensor with a bandwidth of $500 \mathrm{MHz}$ to $3 \mathrm{GHz}$ and centered about $930 \mathrm{MHz}$ was employed to obtain the PD signals. The experiment was carried out on a real threephase $110 \mathrm{kV}$ GIS (ZF10-126) in one tank type in a metallic shield high voltage test hall at Shandong Taikai High Voltage Switchgear Co. Ltd. A brief schematic diagram of testing and detection circuit is shown in Fig. 1.

In Fig. 1, T1 is a column type voltage regulator, T2 is a power frequency testing transformer (YDTCW-1000/

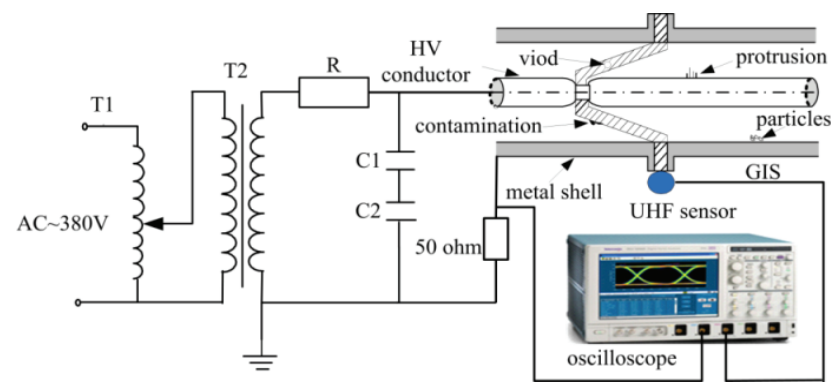

Fig. 1. Schematic diagram of PD detection circuit

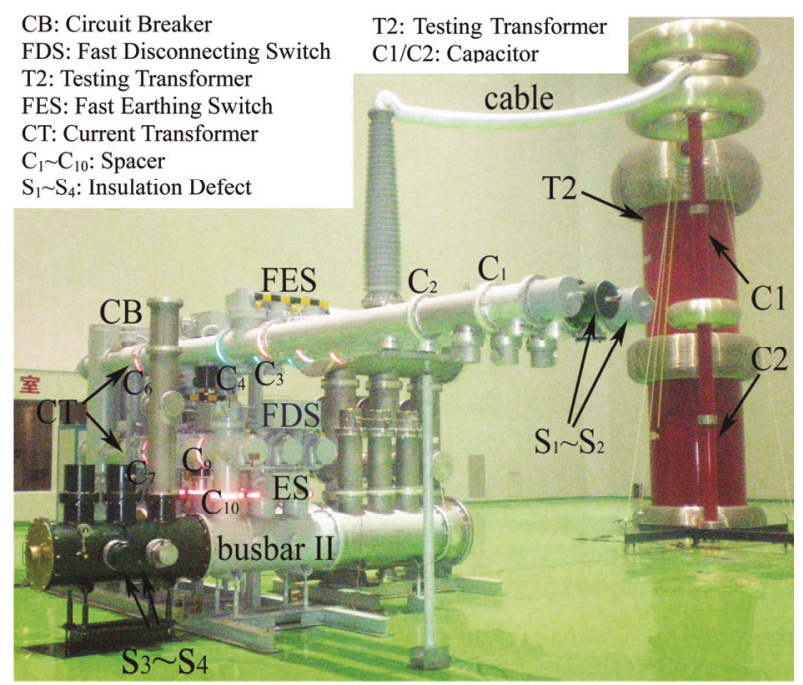

Fig. 2. The main equipment for online detection
$2 \times 500$ ) without partial discharge, $\mathrm{C} 1 / \mathrm{C} 2$ are power frequency capacitor divider \& coupling capacitor (TAWF$1000 / 600), \mathrm{R}$ is a power frequency protecting resistance (GR1000-1/6), and the oscilloscope is Tektronix DPO7104 with a bandwidth of $1 \mathrm{GHz}$ and $20 \mathrm{GS} / \mathrm{s}$ sampling rate. The main equipment is shown in Fig. 2.

Four artificial insulation defects were implemented in the GIS, as shown in Fig. 3. The protrusion defect $S_{1}$ was simulated with a $2 \mathrm{~mm}$ diameter copper wire tied on the $\mathrm{HV}$ conductor with a distance of $40 \mathrm{~mm}$ to the inwall of the chamber (Fig. 3a). The free particles defect $S_{2}$ was simulated in some $2 \mathrm{~mm} * 2 \mathrm{~mm}$ aluminum sheets placed on

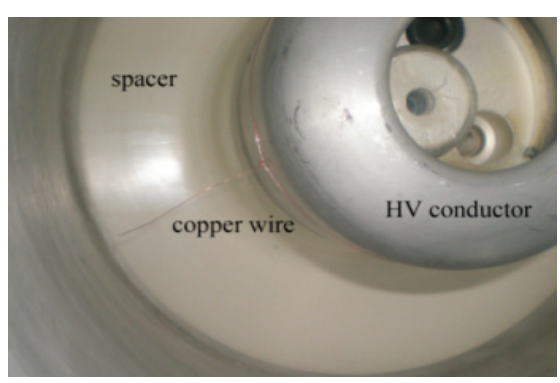

(a) Protrusion $\mathrm{S}_{1}$

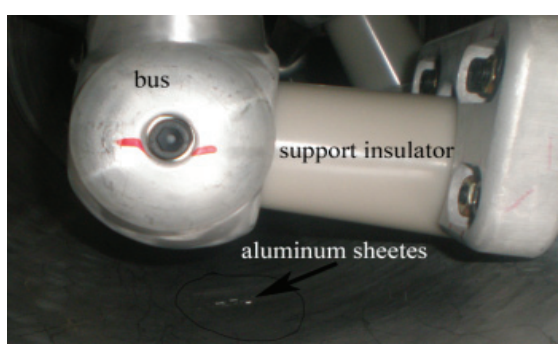

(b) Free particles $\mathrm{S}_{2}$

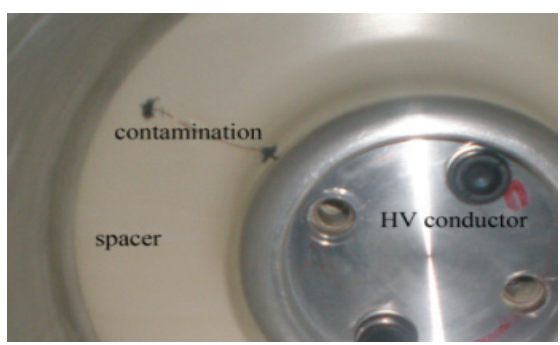

(c) Surface contamination $\mathrm{S}_{3}$
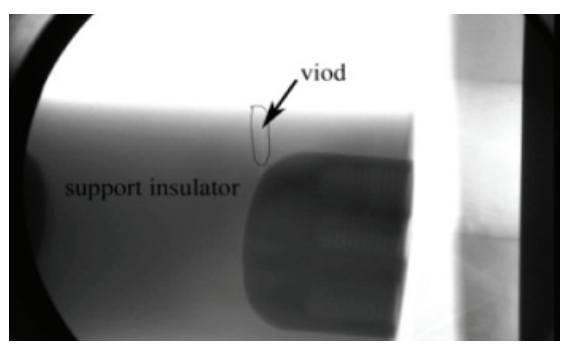

(d) Void $\mathrm{S}_{4}$

Fig. 3. Four artificial defects in a real GIS 
the bottom of the busbar tube (Fig. 3b). The surface contamination defect $\mathrm{S}_{3}$ was simulated with a $2 \mathrm{~mm}$ diameter copper wire with a distance of $15 \mathrm{~mm}$ to the $\mathrm{HV}$ pole and $35 \mathrm{~mm}$ to the inwall of the chamber adhered to the surface of an insulating spacer (Fig. 3c). A bad support insulator with a flaw of about $43 \mathrm{~mm}$ caused by pressure overload was taken as void in insulating spacer defect $\mathrm{S}_{4}$ and (Fig. 3d). Distribution of the four defects is shown in Fig. 2, the protrusion defect $S_{1}$ and surface contamination defect $\mathrm{S}_{3}$ were respectively installed in two separate gas chambers near insulating spacer $C_{1} . S_{1}$ and $S_{2}$ were installed in busbar tube II.

In order to mitigate the effects of attenuation, refraction and reflection, the UHF sensor was placed at spacer $C_{1}$ for detection of $\mathrm{S}_{1}$ and $\mathrm{S}_{3}$ defects, while placed at spacer $\mathrm{C}_{10}$ for $\mathrm{S}_{2}$ and $\mathrm{S}_{4}$ defects.

With the oscilloscope, a single phase resolved partial discharge (PRPD) sample is composed of PD signals in sequential 50 power cycles $(50 \mathrm{~Hz}$ in China). The sampling rate was set at $50 \mathrm{MS} / \mathrm{s}$, and the maximum value of each interval was remained by 2778:1 resampling, which means that a signal data is of 360 points corresponding to a power frequency cycle.

\subsection{Chemical method offline}

When $\mathrm{PD}$ occurs in $\mathrm{SF}_{6}$ gas, a portion of $\mathrm{SF}_{6}$ molecules is decomposed and reacts with $\mathrm{H}_{2} \mathrm{O}$ and $\mathrm{O}_{2}$, which are impurities in $\mathrm{SF}_{6}$. Many kinds of chemical products are generated, such as $\mathrm{SOF}_{4}, \mathrm{SOF}_{2}, \mathrm{SO}_{2} \mathrm{~F}_{2}, \mathrm{SF}_{4}, \mathrm{SO}_{2}, \mathrm{CF}_{4}, \mathrm{CO}_{2}$, $\mathrm{HF}$ [9], and so forth. These decomposition products can indicate $\mathrm{PD}$ in GIS, allowing for the $\mathrm{PD}$ recognition by analyzing $\mathrm{SF}_{6}$ decomposition products.

However, in practical application, it does not allow gas extraction from gas chambers of GIS in service. To simulate the process of $\mathrm{SF}_{6}$ decomposition, an effective simulation assembly was established in the lab. As shown in Fig. 4, artificial defects were placed in the gas chamber filled with pressurized $\mathrm{SF}_{6}$. High voltage was applied to the artificial defects and $\mathrm{PD}$ was generated to decompose $\mathrm{SF}_{6}$, whose decomposition products were detected by a gas chromatograph (Varian CP-3800).

The four corresponding artificial defects were designed for the simulation of offline measurement. Fig. 5(a) illustrates the protrusion defect $\mathrm{S}_{1}$ simulated by a needle-

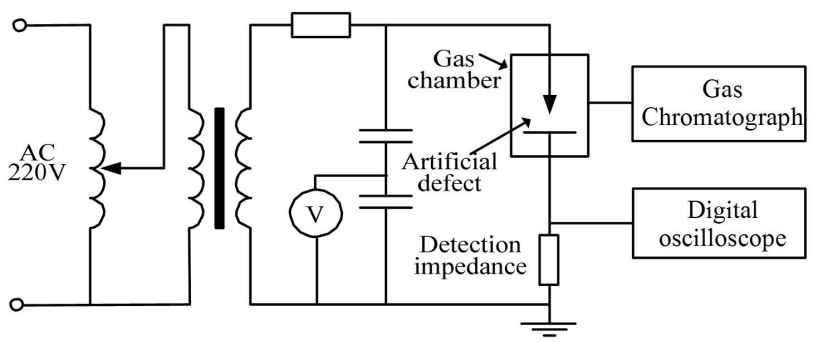

Fig. 4. Schematic diagram of chemical detection system

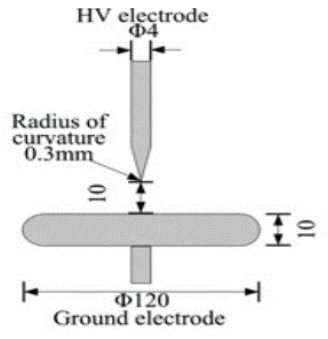

(a)

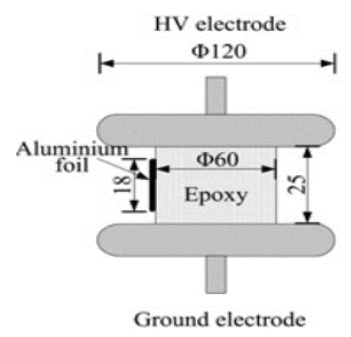

(c)

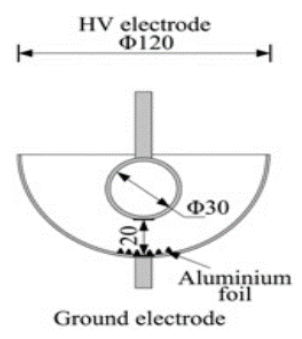

(b)

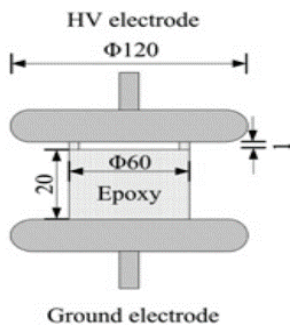

(d)
Fig. 5. Four kinds of artificial defect models (unit: mm)

plane electrode. The particle defect $\mathrm{S}_{2}$ consisted of a sphere electrode, a hemisphere electrode, and copper cuttings that could move freely in the hemisphere electrode, as shown in Fig. 5(b). To simulate the contamination defect $\mathrm{S}_{3}$, some copper cuttings were adhered on the surface of an epoxy block as shown in Fig. 5(c). To create a void defects $\mathrm{S}_{4}$, an epoxy block was inserted between two plane electrodes, as shown in Fig. 5(d). There was a small gap between the epoxy and the upper electrode. To make the simulation more practical, the high-voltage electrodes were made of aluminum and the ground electrodes were made of steel, which is similar to real GIS.

\subsection{Experimental procedure}

To rapidly simulate the development of defect or different serious states, the step stress test method was adopted both for UHF and chemical methods. First, gradually increase the test voltage until the first PD signal occurred. The voltage applied on the defect at this moment was the PD inception voltage. Several test voltages higher than the inception voltage were selected to generate PD signals. For UHF detection, each experiment lasted for $2 \mathrm{~h}$ to obtain the information. And for chemical method each one lasted for $96 \mathrm{~h}$ and the concentrations of four typical decomposition were detected every $4 \mathrm{~h}$ by the gas chromatograph in chemical method.

\subsection{PD signals acquisition}

As shown in Fig. 6, typical PRPD mode of PD signals obtained with the UHF sensor. For each defect, 100 PRPD sample sets were collected respectively.

For chemical detection method under each defect, 72 samples for the concentrations of $\mathrm{SO}_{2} \mathrm{~F}_{2}, \mathrm{SOF}_{2}, \mathrm{CF}_{4}$ and 


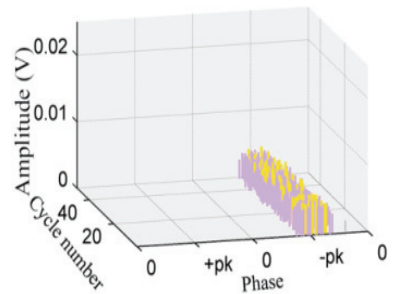

(a)

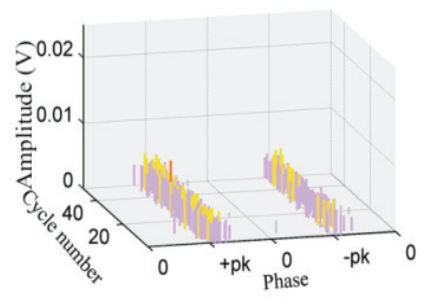

(c)

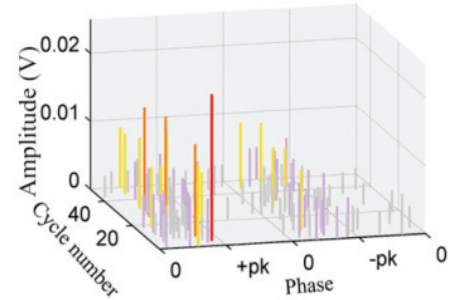

(b)

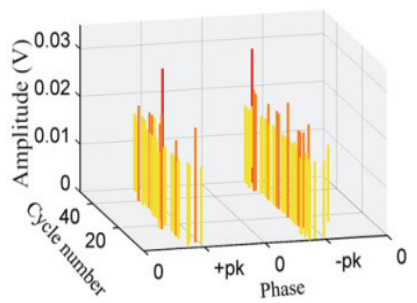

(d)
Fig. 6. PRPD data for (a) Protrusion $\mathrm{S}_{1}$; (b) Free particles $\mathrm{S}_{2}$; (c) Surface contamination $\mathrm{S}_{3}$; (d) Void in insulating spacer $\mathrm{S}_{4}$.

$\mathrm{CO}_{2}$ were detected every $4 \mathrm{~h}$ by the gas chromatograph.

\section{Fault Diagnosis using Single Method}

Conventional clustering algorithm, such as $\mathrm{NN}$ and SVM, is a kind of hard partition algorithm, which is not able to directly differentiate defect types due to the complex features of PD signals. As an unsupervised classification, fuzzy C-means (FCM) clustering algorithm $[15,16]$ can be used to examine the intermediary nature of the sample, to reflect the PD natural attributes objectively, and even to classify the samples with a fuzzy property. The algorithm is applicable for diagnosis with UHF method as well as the chemical method.

\subsection{Basic FCM theory}

The theory of FCM is as follows.

Let $R$ be the set of real numbers and $R^{s}$ be the $d$ dimensional vector space over the real numbers. The sample set is $X=\left\{x_{1}, x_{2}, \ldots, x_{n}\right\} \in R^{s}$, and $c$ represents the number of categories. The objective function of FCM is shown in Eq. (1).

$$
\min J(U, V)=\sum_{i=1}^{n} \sum_{k=1}^{c}\left(u_{i k}\right)^{m}\left(d_{i k}\right)^{2}
$$

where $V=\left[v_{k}\right]_{c \times s}$ is the cluster matrix, $v_{k}$ is the cluster of class $k, 1 \leq k \leq c \leq n, U=\left[u_{i k}\right]_{s \times c}$ represents the membership degree matrix, $u_{i k}$ is the membership degree of $x_{i}$ belonging to class $k ; u_{i k} \in[0,1], \sum_{k=1}^{c} u_{i k}=1, \quad m \in[1, \infty]$ is the weighting coefficient; and $d_{i k}$ represents the distance between sample $x_{i}$ and the cluster $v_{k}$ :

$$
\left(d_{i k}\right)^{2}=\left\|x_{i}-v_{k}\right\|^{2}
$$

To find the optimal $U=\left[u_{i k}\right]$ and $v_{k}$ that minimize $J(U, V)$, the objective function can be solved by the Lagrange multiplier:

$$
F=\sum_{k=1}^{c}\left(u_{i k}\right)^{m}\left(d_{i k}\right)^{2}+\lambda\left(\sum_{k=1}^{c} u_{i k}-1\right)
$$

The optimal condition of Eq. (3) is:

$$
\left\{\begin{array}{c}
\frac{\partial F}{\partial \lambda}=\left(\sum_{k=1}^{c} u_{i k}-1\right)=0 \\
\frac{\partial F}{\partial \lambda}=\left[m\left(u_{i k}\right)^{m-1}\left(d_{i k}\right) 2-\lambda\right]=0
\end{array}\right.
$$

By solving Eq. (4), we can get $u_{i k}$ and $v_{k}$ shown in Eq. (5-6):

$$
\begin{gathered}
u_{i k}=\sum_{j=1}^{c}\left(\frac{d_{i k}}{d_{i j}}\right)^{\frac{2}{1-m}},(j=1,2, \cdots, c) \\
v_{k}=\frac{\sum_{i=1}^{n}\left(u_{i k}\right)^{m} x_{i}}{\sum_{i=1}^{n}\left(u_{i k}\right)^{m}}
\end{gathered}
$$

FCM clustering algorithm is presented as follows:

1) Determine $X, m, c$ and generate the initial membership matrix $U^{(0)}=\left[u^{(0)}{ }_{i k}\right]$ randomly;

2) Compute the cluster center of each class by Eq. (6);

3) Calculate the new membership degree matrix by Eq. (5);

4) If the distance between the membership degree matrixes of the two successive iterations satisfies $\left\|U^{l}-U^{l-1}\right\| \leq 10^{-4}$, stop the calculation. Otherwise, continue the calculation from step 3).

\subsection{Fault diagnosis with UHF method}

With comparison of typical PRPD spectrums shown in Fig.6, it can be found that PRPD for either $S_{1}$ or $S_{2}$ show strong regularity with quite similar spectrums as applied voltage changes and PD develops, while the other two are dissimilar and fickle. For field application, it's hard for the engineer to get the diagnostic results directly from the analysis of PRPD.

For better diagnostic results, feature extraction needs to be done first. A classic feature extraction method based on 
Table 1. Extracted features of PRPD

\begin{tabular}{|c|c|}
\hline Index & Features \\
\hline $1-6$ & $\begin{array}{c}S k_{m}^{+} S k_{m}^{-} S k_{m} \\
S k_{n}^{+} S k_{n}^{-} S k_{n} \\
\end{array}$ \\
\hline $7-12$ & $\begin{array}{c}K u_{m}{ }^{+} K u_{m}^{-} K u_{m} \\
K u_{n}^{+} K u_{n}^{-} K u_{n}\end{array}$ \\
\hline $13-14$ & $Q m Q n$ \\
\hline $15-16$ & $c c_{m} c c_{n}$ \\
\hline
\end{tabular}

* $S_{k}$ is skewness, $K_{u}$ is kurtosis, $Q$ is discharge factor, $c c$ is modified cross correlation factor; $m$ is for amplitude, $n$ is for PD number; + respectively means the positive and negative half of power frequency cycle.

Table 2. Diagnostic results with UHF method

\begin{tabular}{|c|c|c|c|c|c|c|}
\hline \multirow{2}{*}{$\begin{array}{c}\text { PD } \\
\text { Types }\end{array}$} & \multicolumn{4}{|c|}{ Predicted results } & \multirow{2}{*}{ Total } & \multirow{2}{*}{ Accuracy } \\
\hline & $\mathrm{S}_{1}$ & $\mathrm{~S}_{2}$ & $\mathrm{~S}_{3}$ & $\mathrm{~S}_{4}$ & & \\
\hline $\mathrm{S}_{1}$ & 92 & 5 & 2 & 1 & 100 & $92 \%$ \\
\hline $\mathrm{S}_{2}$ & 1 & 4 & 5 & 90 & 100 & $90 \%$ \\
\hline $\mathrm{S}_{3}$ & 5 & 9 & 81 & 5 & 100 & $81 \%$ \\
\hline $\mathrm{S}_{4}$ & 5 & 6 & 9 & 80 & 100 & $80 \%$ \\
\hline Total & & & & & & $85.8 \%$ \\
\hline
\end{tabular}

statistical theory [17] was employed, and all the extracted features are shown in Table 1.

As shown in Table 1, the complex features of PRPD data are of 16-dimension, making it difficult to classify the similarity between the categories and objects. Thus the FCM algorithm is introduced to find the intermediary nature of the PRPD data samples. To avoid attributes existed in greater numeric ranges which may dominate over those in smaller numeric ranges and to limit numerical difficulties, typical normalization method was applied. Take the normalization result as the input variable $X$, set $c=4, m=2$. The diagnostic results with UHF method based on FCM clustering algorithm are shown in Table 2.

It can be easily concluded that the overall accuracy of FCM diagnosis with UHF method is $85.8 \%$, including a higher accuracy of $\mathrm{S}_{1}$ and $\mathrm{S}_{2}$ defect. As for surface contamination defect $S_{3}$ and void in spacer $S_{4}$, the results show a less satisfactory accuracy due to the variation on shapes, size, ageing degree of the two defects.

\subsection{Fault diagnosis with chemical method}

Obviously, the concentration data of $\mathrm{SF}_{6}$ decomposition products under four typical defects have no strict boundary, the fuzzy property of which FCM can deal with. Similar to the three-ratio method widely used in the fault diagnosis of transformers [18, 19], selecting concentration ratio of $\mathrm{SF}_{6}$ decomposition products as the characteristic quantity may be a more preferred method.

After analyzing the mechanism of chemical reaction process [20], three representative concentration ratios with distinct values and clear physical significance are chosen as features for diagnosis with chemical method. $\mathrm{c}\left(\mathrm{SOF}_{2}\right) /$ $\mathrm{c}\left(\mathrm{SO}_{2} \mathrm{~F}_{2}\right)$ is the indication of discharge energy of $\mathrm{PD}$ for they are the main products when $\mathrm{SF}_{6}$ is decomposed under

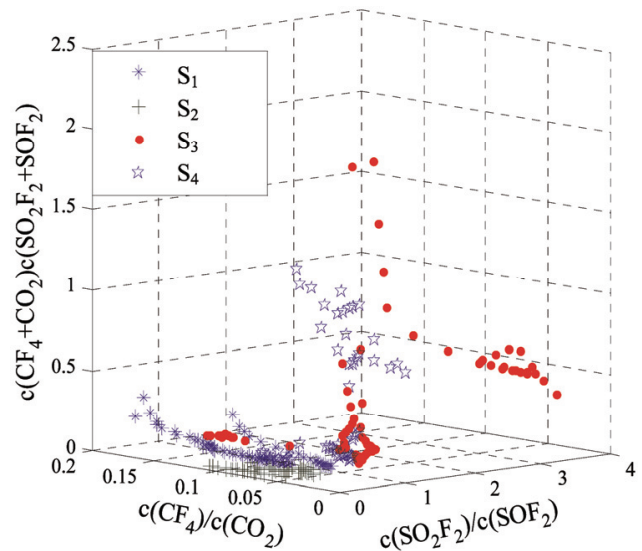

Fig. 7. Distribution of concentration ratio samples

Table 3. Diagnostic results with chemical method

\begin{tabular}{|c|c|c|c|c|c|c|}
\hline \multirow{2}{*}{$\begin{array}{c}\text { PD } \\
\text { Types }\end{array}$} & \multicolumn{4}{|c|}{ Predicted results } & \multirow{2}{*}{ Total } & \multirow{2}{*}{ Accuracy } \\
\hline & $\mathrm{S}_{1}$ & $\mathrm{~S}_{2}$ & $\mathrm{~S}_{3}$ & $\mathrm{~S}_{4}$ & & \\
\hline $\mathrm{S}_{1}$ & 58 & 6 & 6 & 2 & 72 & 6 \\
\hline $\mathrm{S}_{2}$ & 5 & 56 & 7 & 4 & 72 & 56 \\
\hline $\mathrm{S}_{3}$ & 3 & 7 & 58 & 4 & 72 & 7 \\
\hline $\mathrm{S}_{4}$ & 5 & 6 & 9 & 52 & 72 & 6 \\
\hline Total & & & & & & $77.8 \%$ \\
\hline
\end{tabular}

$\mathrm{PD}, \mathrm{c}\left(\mathrm{CF}_{4}\right) / \mathrm{c}\left(\mathrm{CO}_{2}\right)$ indicates structures of different defects for they are the reaction products of carbon atoms in the high temperature region of the electrode and epoxy resin with $\mathrm{O}_{2}$, and $\mathrm{c}\left(\mathrm{CF}_{4}+\mathrm{CO}_{2}\right) / \mathrm{c}\left(\mathrm{SOF}_{2}+\mathrm{SO}_{2} \mathrm{~F}_{2}\right)$ has a positive correlation with the degradation degree of insulation materials or electrodes. In this paper, 72 samples of SF6 decomposition products were collected from chemical offline experiments on four typical defects respectively. The distribution of the samples in the three-dimensional space is shown in Fig. 7.

Take the three concentration ratio as the input variable $X$, set $c=4, m=2$. The diagnostic results with chemical method based on FCM clustering algorithm are shown in Table 3.

The overall accuracy of diagnostic results with chemical method is $77.8 \%$. The diagnostic result indicates the lowest accuracy for the particle defect S2 because the particle can move freely under the electric field resulting more randomness. As shown above, diagnostic performance for different defects with UHF and chemical methods differ greatly, which may cause misclassification. Especially when prior knowledge is lacked in field application, getting a conclusion is almost impossible.

\section{Fault Diagnosis Based on Joint Detection Method and DS Evidence Theory}

In order to enhance the integrality, accuracy, fault tolerance and availability of the diagnostic results, a complete perceptive description and interpretation of the objects (PD) should be constructed according to the internal rules and 
relation. By employing DS evidence theory, the incomplete, uncertainty, fuzzy even conflicting information can be processed to obtain more reliable information, resulting in better diagnostic results. The synthetic diagnosis method based on DS evidential theory and the joint electrochemical method is presented in this paper.

\subsection{Evidence Theory}

In DS evidence theory, the belief function $(\mathrm{Bel})$ is introduced to measure uncertainty which satisfies weak axiom of probability and the plausibility function $(P l)$ is introduced to deal with uncertainty which is caused by "Do not know"; compared with other method, the evidence theory can distinguish between uncertainty and "Do not know" when the prior probability is difficult but must be given, so the evidence theory has great flexibility. The DS theory defines a frame of discernment, denoted as $\Theta$. A basic hypothesis (singleton) in $\Theta$ is denoted as $A_{i}$. The hypotheses in $\Theta$ are assumed to be mutually exclusive and exhaustive. All the possible results are corresponding to protrusion defect, free particles defect, void in insulating spacer defect, surface contamination defect and the uncertainty $\theta$.

$$
\Theta=\left\{A_{1}, A_{2}, A_{3}, A_{4}, \theta\right\}
$$

The impact of each distinct piece of evidence on the subsets of $\Theta$ is represented by a function called a basic probability assignment (BPA). The BPA function must satisfy the following formulae.

$$
m(\phi)=0 \text { and } \sum_{A \subseteq \Theta} m(A)=1
$$

$$
\text { Here, } \quad m_{i}\left(A_{j}\right)=\alpha_{i} * \frac{O_{j}}{\sum_{j=1}^{j=4} O_{j}} \text { refers to the degree of belief }
$$

held by an observer regarding a certain defect and there are two pieces of evidence from UHF method and chemical method, and four types of defects with uncertainty $\theta$. Define $m_{i}\left(A_{j}\right)=\alpha_{i} * \frac{O_{j}}{\sum_{j=1}^{j=4} O_{j}}$ as the BPA function of the $i$-th evidence for the $j$-th defect, then it can be calculated in formula as follows:

$$
\begin{gathered}
m_{i}\left(A_{j}\right)=\alpha_{i} * \frac{O_{j}}{\sum_{j=1}^{j=4} O_{j}} i=1,2 ; j=1,2,3,4 \\
m_{i}(\theta)=1-\alpha_{i}
\end{gathered}
$$

$O_{j}$ represents the $j$ th output of the neural network, obviously, $\sum_{m=1}^{4} m_{i}\left(A_{j}\right)+m_{i}(\theta)=1$, satisfying the definition
of function $m$.

When all the BPA functions have been calculated with the combination rules, PD types of the unknown insulation defect can be judged with the following three rules.

Rule I, $m\left(A_{\max 1}\right)=\max \left\{m\left(A_{i}\right), A_{i} \subset \Theta\right\}$, meaning that the judged PD type should have a maximum BPA.

Rule II, $m\left(A_{\max 1}\right)=m(\theta)$; meaning that the BPA of the judged PD type should be larger than the uncertainty $\theta$.

Rule III, $m\left(A_{\max 1}\right)-m\left(A_{\max 2}\right)>\varepsilon$;

Where, $m\left(A_{\max 2}\right)$ is the second largest $\mathrm{BPA}$, and $\varepsilon$ is a threshold larger than 0 . The value of $\varepsilon$ depends on the attributes of information samples, practical application. Only when the judged PD type satisfies all the three rules can the fusion decision-making system make the final discriminant of the PD type with the largest BPA.

\subsection{Fault diagnosis with joint electro-chemical method}

To evaluate the performance of the proposed diagnosis system for tests with data samples, PD samples with joint method are selected randomly for each defect. In a practical application, $\operatorname{Bel}(A), \operatorname{Pl}(A)$ or a value between them can be considered as the reliability coefficient. Here, let $\alpha$ represent the average diagnostic accuracy. The diagnostic results shown in Table 4 are obtained based on DS theory as shown above.

Table 4. Diagnostic results with joint method

\begin{tabular}{c|c|c}
\hline Defect & Correct / Undetermined /Fault & Accuracy \\
\hline$S_{1}$ & $72 / 0 / 0$ & $100 \%$ \\
\hline$S_{2}$ & $69 / 2 / 1$ & $96 \%$ \\
\hline$S_{3}$ & $71 / 0 / 1$ & $99 \%$ \\
\hline$S_{4}$ & $71 / 1 / 0$ & $99 \%$ \\
\hline Total & & $98.26 \%$ \\
\hline
\end{tabular}

After fusing the two diagnostic results based on DS evidence theory, the new diagnostic method has a higher accuracy than the other two, with diagnostic result for each defect type reaching $96 \%$ at least. Furthermore, it's found that there's few undetermined samples. Maybe the information provided by the two evidences produces serious conflicts, which results in no final decision or even false diagnosis. The introduction of uncertainty $\theta$ in DS evidence theory makes the diagnostic results more reliable, while the common methods adopts the competitive output rules which simply make a decision between "belong to" or "not", reducing the reliability. In field application where the defects always are not included in the database due to the lack of prior knowledge, the uncertainty $\theta$ is of great practical significance.

Actually, another possible fault diagnostic way can be obtained with the characteristic quantity of joint electrochemical method directly combined into a 19-dimensional 
Table 5. Comparison of FCM and FCM-DS

\begin{tabular}{c|c|c|c|c|c}
\hline \multirow{2}{*}{ Method } & \multicolumn{4}{|c|}{ Diagnostic accuracy } & \multirow{2}{*}{ Time-taken } \\
\cline { 2 - 5 } & $\mathrm{S}_{1}$ & $\mathrm{~S}_{2}$ & $\mathrm{~S}_{3}$ & $\mathrm{~S}_{1}$ & \\
\hline FCM & $93 \%$ & $85 \%$ & $86 \%$ & $93 \%$ & 205 \\
\hline FCM-DS & $100 \%$ & $96 \%$ & $99 \%$ & $100 \%$ & 96 \\
\hline
\end{tabular}

features after the normalization, which can be taken as the input of FCM algorithm. Whereas, more extracted features bring in computational complexity of the system and slow down the speed of data processing. The diagnostic results and the time-taken for FCM and FCM-DS method are shown in Table 5.

From Table 5 above, the diagnostic accuracy by taking the total 19-dimensional features as input vector of FCM is no enhanced remarkably compared to the results shown in Table 2 and Table 3. Firstly, the competitive output rules of FCM cause information underprivileged samples lost. Secondly, the comprehensive input makes FCM hard to identify the redundancy and validity of the information. In the end, the higher dimensional features increase the difficulty of recognition, leading to a longer time-taken as well. As for the FCM-DS method which combines the outputs of two FCM classifiers based on DS theory, the original features are processed in parallel, and hence effectively reducing the calculation complexity and accelerating the computation speed.

\section{Conclusion}

The precision and reliability of fault diagnosis with PD measurement plays an essential role in condition assessment of GIS, which is mostly determined by both data source and data processing ability. In this paper, a more complete interpretation of PD data is formed with the proposed joint electro-chemical detection method, and decision making of classifying PD types is also solved by DS evidence theory by fusing two diagnostic results with UHF and chemical method respectively. The performance of fault diagnosis with every single detection alongside the joint detection method are described and compared carefully. Diagnostic results under direct combination of two kinds of features are also compared with that with fusion of FCM results. Obtained results show that the proposed diagnosis system based on joint detection method and DS evidence theory has significantly improved the accuracy with a more credible decision, which significantly reduces the possibility of erroneous diagnosis and enhances the fault tolerance. To reduce the calculation complexity, the original features are processed with FCM algorithm in parallel, resulting in accelerated the computation speed and a shorter time-taken. Last, but not least, the ability of handling uncertainty $\theta$ makes it promising way to give a relatively credible prediction of some uncertain defect in filed application without sufficient prior knowledge.
In brief, this paper investigates a new fault diagnosis system to resolve the problems of incomplete PD data source and the reliability of data processing. Results of tests on the four typical defects show that it provides a promising way to make a more accurate decision with more practical application for condition assessment of GIS.

\section{Acknowledgements}

The research work has been funded by the National Natural Science Foundation of China (Grant No. 5177181) and National Basic Research Program of China (Program 973, Grant No.2009CB724506) and. The authors sincerely thank the granting agency.

\section{References}

[1] Maren Istad and Magne Runde, "Thirty-six Years of Service Experience with a National Population of Gas Insulated Substations," IEEE Transaction on Power Delivery, vol. 25, no. 4, pp. 2248-2454, Jun. 2010.

[2] R. Bartnikas., "Partial Discharges: Their Mechanism, Detection and Measurement," IEEE Transaction on Dielectrics and Electrical Insulation, vol. 9, no. 2, pp.763-808, Oct.2002.

[3] N.C. Sahoo, M.M.A. Salama, and R. Bartnikas, "Trends in Partial Discharge Pattern Classification: a survey," IEEE Transaction on Dielectrics and Electrical Insulation, vol. 12, no. 2, pp. 248-264, Apr. 2005.

[4] Cheol-Hwi Ryu, Seung-Yong Jung, Ja-Yoon Koo and Man-Seung Yeon, "An Application of the Novel Techniques Detecting Partial Discharge Employable to GIS Using Optical Sensor," Journal of Electrical Engineering \& Technology, vol. 2, no. 3, pp. 396 400, Sep. 2007

[5] Seoung-Yong Jung, Cheol-Hwi Ryu, Yun-Sok Lim, Ja-Ho Lee and Ja-Yoon Koo, "Discrimination of Insulation Defects in a Gas Insulated Switchgear (GIS) by use of a Neural Network Based on a Chaos Analysis of Partial Discharge (CAPD)," Journal of Electrical Engineering \& Technology, vol. 2, no. 1, pp. 118 122, Mar.2007

[6] Biswas S, Dey D, Chatterjee B, et al. "An approach Based on Rough Set Theory for Identification of Single and Multiple Partial Discharge source," International Journal of Electrical Power, vol.45, no.1, pp. 163-174, Feb.2013.

[7] Mohammad Shukri Hapeez, Ngah Ramzi Hamzah, Habibah Hashim and Ahmad Farid Abidin, "A New Reliable Algorithm for Identifying Types of Partial Discharge Detected through Ultrasonic Emission," Journal of Electrical Engineering Technology, vol. 9 
no. 1, pp.259-267, Jan.2014

[8] Fan Liu, Ju Tang, Yilu Liu, "Mathematical Model of Influence of Oxygen and Moisture on Feature Concentration Ratios of $\mathrm{SF}_{6}$ Decomposition Products," IEEE PES General Meeting, San Diego, U.S.A, Jul.2012

[9] Ju Tang, Fan Liu, Xiangxing Zhang, Qinghong Meng and Jiabin Zhou, "Partial Discharge Recognition through an Analysis of $\mathrm{SF}_{6}$ Decomposition Products part 1: Decomposition Characteristics of $\mathrm{SF}_{6}$ under Four Different Partial Discharges," IEEE Transactions on Dielectrics and Electrical Insulation, vol. 19, no. 1, pp. 29-36, Feb. 2012.

[10] Ju Tang, Fan Liu, Qinghong Meng, Xiaoxing Zhang and Jiagui Tao, "Partial Discharge Recognition through an Analysis of SF6 decomposition products part 2: feature extraction and decision tree-based pattern recognition," IEEE Transactions on Dielectrics and Electrical Insulation, vol. 19, no. 1, pp. 37-44, Feb. 2012.

[11] Venkatesh S, Gopal S, "Robust Heteroscedastic Probabilistic Neural Network for multiple source partial discharge pattern recognition - Significance of outliers on classification capability," Expert System Application, vol.38, no.9, pp.501-514, Nov.2011.

[12] Hao L, Lewin P L, Tian Y, et al., "Partial discharge identification using a support vector machine," Annual Report Conference on Electrical Insulation and Dielectric Phenomena, Nashville, USA, Oct. 2005.

[13] Shafer G, A mathematical theory of evidence: Princeton University Press, Princeton, 1976, pp. 8-48

[14] Ph. Smets, "Belief functions on real numbers", International Journal of Approximate Reason, vol. 40, no. 3, pp. 181-223, Mar. 2005.

[15] Mordjaoui Mourad and Boudjema Bouzid, "Dynamic Hysteresis Model Based on Fuzzy Clustering Approach," Journal of Electrical Engineering Technology, vol. 7, no. 6, pp.884-890, Nov.2012

[16] Yu Jian and Yang Miin-Shen, "Optimality test for generalized FCM and its application to parameter selection," IEEE Transactions on Fuzzy Systems, vol. 13, no. 1, pp. 164-176, Apr.2005.

[17] Ju Tang, Jiagui Tao, Xiangxing Zhang, Fan Liu, "Multiple SVM-RFE for feature subset selection in partial discharge pattern recognition," International Review of Electrical Engineering, vol. 7, no. 4, pp. 5240-5246, Aug. 2012.

[18] Rahmat-Allah Hooshmand and Mahdi Banejad, "Fuzzy Logic Application in Fault Diagnosis of Transformers Using Dissolved Gases," Journal of Electrical Engineering \& Technology, vol. 3, no. 3, pp. 293 299, Sep. 2008

[19] Nitin K. Dhote and J. B. Helonde, "Improvement in Transformer Diagnosis by DGA using Fuzzy Logic," Journal of Electrical Engineering \& Technology, vol.
9, no. 2: 615-621, Mar.2014.

[20] F. Y. Chu, "SF6 Decomposition in Gas-insulated Equipment," IEEE Transactions on Electrical Insulation, vol. 21, no.5, pp. 693-725, Oct. 1986.

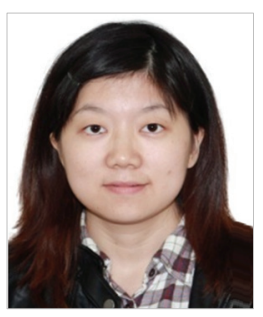

Liping Li She received the B.Sc. degree in Electronic Science and Technology from Harbin Institute of Technology, China and the M.Sc. degree in Optical Engineering from Tianjin University of Technology, China. She is currently pursuing the $\mathrm{PhD}$ degree in Chongqing University, Chongqing, China. Her research interests include partial discharge measurement, pattern recognition, condition assessment of GIS.

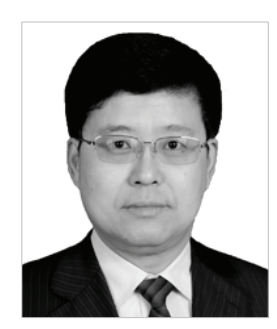

Ju Tang He received the M.Sc. and Ph.D. degrees from Chongqing University, Chongqing, China. Currently, he is the Dean of School of Electrical Engineering in Wuhan University and the Chief Scientist who is presiding over a National Basic Research Program of China (973 Program) (2009 CB724506). At present, he is involved in high-voltage equipment online monitoring, fault diagnosis, signal processing, simulation analysis, and pattern recognition.

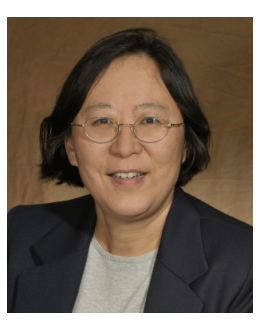

Yilu Liu She received her M.S. and $\mathrm{Ph} . \mathrm{D}$. degrees from the Ohio State University, Columbus, in 1986 and 1989. She received the B.S. degree from Xian Jiaotong University, China. Currently, she is the UT-ORNL Governor's Chair at the University of Tennessee, Knoxville and Oak Ridge National Laboratory (ORNL). She is also the deputy Director of the DOE/NSF engineering research center CURENT. Her current research interests include power system wide-area monitoring and control, large interconnection-level dynamic simulations, electromagnetic transient analysis, and power transformer modeling and diagnosis. 